\title{
Overexpression of Lin 28 inhibits the proliferation, migration and cell cycle progression and induces apoptosis of BGC-823 gastric cancer cells
}

\author{
HU SONG ${ }^{1,2}$, WEI XU ${ }^{1}$, JUN SONG ${ }^{1}$, YONG LIANG ${ }^{1}$, WEI FU ${ }^{1}$, XIAO-CHENG ZHU ${ }^{1}$, \\ CHAO LI ${ }^{1}$, JUN-SHENG PENG ${ }^{3}$ and JUN-NIAN ZHENG ${ }^{2}$ \\ ${ }^{1}$ Department of Gastrointestinal Surgery, The Affiliated Hospital of Xuzhou Medical College, Xuzhou, \\ Jiangsu 221002; ${ }^{2}$ Jiangsu Province Tumor Biological Therapy Institute, Xuzhou, Jiangsu 221000; \\ ${ }^{3}$ Department of Gastrointestinal Surgery, The Sixth Affiliated Hospital (Gastrointestinal and \\ Anal Hospital), Sun Yat-sen University, Guangzhou, Guangdong 510655, P.R. China
}

Received June 4, 2014; Accepted August 7, 2014

DOI: $10.3892 /$ or.2014.3674

\begin{abstract}
Lin 28 plays important roles in the development, maintenance of pluripotency and progression of various types of cancers. Lin 28 represses the biogenesis of let-7 microRNAs and is implicated in both development and tumorigenesis. Oncogenic regulation of let-7 microRNAs has been demonstrated in several human malignancies, yet their correlation with Lin 28 has not yet been studied in gastric cancer. Therefore, in the present study, we explored the possible mechanisms involved in the effects by Lin 28 on the proliferation, migration, cell cycle arrest and apoptosis in gastric cancer cells via alteration of let-7 miRNA. The expression levels of Lin28 and let-7 were detected by real-time PCR in gastric cancer cell lines in vitro. Lin 28 was overexpressed in the BGC-823 cells via lentiviral transfection, and let-7 expression was assessed. Cell proliferation and migration capabilities were investigated by MTT and Transwell assays, while cell cycle distribution and the apoptosis rate were detected using flow cytometry. The expression of Lin 28 was moderately expressed in the GES cells while underexpressed in the BGC-823, SGC-7901 and HGC-27 cells. Let-7a miRNA was highly expressed in the GES, BGC-823, SGC-7901 and HGC-27 cells. Overexpression of Lin28 was inversely correlated with the downregulated expression of let-7a, and markedly suppressed the proliferation, migration, cell cycle progression and induced apoptosis in the BGC-823 cells. These findings demonstrated that overexpression of Lin28 can suppress the biological behavior of gastric
\end{abstract}

Correspondence to: Dr Jun-Nian Zheng, Jiangsu Province Tumor Biological Therapy Institute, The Affiliated Hospital of Xuzhou Medical College, 99 Huaihai Road, Xuzhou, Jiangsu 221002, P.R. China E-mail: zhengjunnian@126.com

Key words: Lin28, let-7a, gastric cancer, proliferation, apoptosis cancer in vitro, and let-7 miRNA may play an important role in the process. We suggest that Lin 28 may be a candidate predictor or an anticancer therapeutic target for gastric cancer patients.

\section{Introduction}

Lin28 is a conserved RNA binding protein that was originally described as an indispensable regulator of developmental timing in Caenorhabditis elegans (1). Mammals have two homologs, Lin28A and Lin28B. In mammals, Lin28A, often called Lin28, is ubiquitously expressed in early embryonic stages and was shown as one of the four factors that convert fibroblasts into induced pluripotent stem (iPS) cells (2). Lin28 is readily expressed in embryos and embryonic stem cells, yet it is either undetectable or its expression remains at low levels in normal adult tissues, suggesting that Lin 28 may play a critical role in cell proliferation and/or differentiation during embryonic development $(3,4)$. Recently, Lin 28 was confirmed to possess the ability to facilitate the reprogramming of human somatic cells to iPS cells (5).

Furthermore, Lin28A/B promote malignant transformation, and their expression is associated with advanced stages of numerous types of tumors, including hepatocarcinoma, nephroblastoma, ovarian carcinoma and germ cell tumors $(6,7)$. It is highly expressed in various tumors, such as hepatocellular carcinoma and colorectal cancer (7-9). Overexpression of Lin28 has been shown to promote cancer cell proliferation (10). High expression of Lin28 is a poor prognostic factor in hepatocellular cancer and it is significantly associated with lymph node metastasis in colorectal cancer. These features make Lin 28 an attractive target for antibody-based therapy. Therefore, understanding the occurrence and expression status of Lin28 has important implications in the prognosis and treatment of cancer.

At the molecular level, Lin28 acts as a suppressor of let-7 microRNA biogenesis (11-14). Lin28 and its homolog, Lin28B have been known to regulate all let-7 family members 
through a maturation process and cellular differentiation (15). Lin28 was reported to act as a transacting regulator which binds let-7 pre-miRNA to block its maturation in embryonic stem cells (14). This particular function of let-7 was used in the process of establishing iPS cells from human fibroblasts in order to enhance the efficiency of cellular formation (16). A number of studies have also suggested an association between Lin28 and let-7, with particular emphasis upon their involvement in cellular processes such as differentiation and proliferation $(13,14)$.

However, a correlation between Lin28 and let-7 in gastric cancer cells has not yet been confirmed. In the present study, we examined the expression of Lin 28 and let-7 in gastric cancer cell lines and investigated the effect of lentiviral-mediated Lin 28 overexpression on proliferation, migration, cell cycle progression and apoptosis of gastric cancer cells in vitro.

\section{Materials and methods}

Cell culture. The human gastric cancer cell lines BGC-823, SGC-7901 and HGC-27 and the gastric mucosal cell line GES were provided by Jiangsu Key Laboratory of Biological Cancer Therapy, Xuzhou Medical College, China. All cell lines were routinely maintained in RPMI-1640 medium (Gibco, Garlsbad, CA, USA) supplemented with $10 \%$ fetal bovine serum (FBS) (Invitrogen, USA), $2 \mathrm{mM}$ glutamine, $100 \mathrm{U} / \mathrm{ml}$ of penicillin and $100 \mathrm{mg} / \mathrm{ml}$ streptomycin (all from Sigma, St. Louis, MO, USA) at $37^{\circ} \mathrm{C}$ with $5 \% \mathrm{CO}_{2}$.

Recombinant lentivirus construction. The human Lin 28 gene fragments were amplified by polymerase chain reaction (PCR) technology. The primers for PCR were synthesized as follows (GeneChem Co., Ltd., Shanghai, China): Lin28 (6596-2)-P1, GAGGATCCCCGGGTACCGGTCGCCACCATGGGCTCC GTGTCCAACCAG and Lin28 (6596-2)-P2, TCCTTGTA GTCCATACCATTCTGTGCCTCCGGGAGCAG. The lentiviral vector Ubi-MCS-GFP (GeneChem) was digested by the restriction enzyme $A g e \mathrm{I}$. Then Lin28 gene fragments were ligated into the GV287 lentiviral vector Ubi-MCS-GFP. The primer, KL6596-P3 (CCCAGTGGATGTCTTTGTGC) located in the coding sequence of the Lin 28 gene was used in PCR to identify positive transformants. Positive clones, as confirmed by PCR, were chosen for sequencing. Recombinant lentiviruses, which coexpress enhanced green fluorescent protein (GFP) and the Lin 28 sequence, were produced by $293 \mathrm{~T}$ cells following the co-transfection of Ubi-MSC-EGFP-Lin28 and the packaging plasmids pHelper 1.0 and pHelper 2.0 (GeneChem) using Lipofectamine 2000 (Invitrogen). Western blot analysis was employed to confirm the overexpression of Lin28A in the transfected 293T cells as described above. Lentiviral-expressing green GFP was generated as a control. The virus titer was detected by quantitative real-time PCR after concentrating and harvesting the viral supernatant.

Transfection. BGC-823 cells in log phase were cultured in 6 -well plates at a density of $5 \times 10^{4}$ cells/well and transfected with Lin28-GFP vectors (Lin28 overexpression group) or GFP vectors (expressing GFP) at a multiplicity of infection (MOI) of 20 in serum-free medium. After incubation at $37^{\circ} \mathrm{C}$ for $16 \mathrm{~h}$, the transduction medium was replaced with fresh
DMEM + 10\% FBS. Lin28A-BGC-823 cells were genetically engineered with a recombinant lentivirus coexpressing EGFP and Lin28 and used as the overexpression (OE) group, and GFP-BGC-823 cells were manipulated with a lentivirus expressing GFP and were used as the negative control (NC) group. BGC-823 cells without intervention served as the normal control (CON) group and cultured in the same manner as described above. After 3 days of transfection, GFP expression was observed using fluorescence microscopy (MicroPublisher 3.3 RTV; Olympus, Japan).

RT-PCR assay. RNAs from the different groups were extracted with TRIzol reagent (Invitrogen) and treated with DNase (Tiangen Biotech, Beijing, China). cDNA was synthesized from $2 \mu \mathrm{g}$ of total RNA according to the manufacturer's instructions in a total volume of $10 \mu \mathrm{l}$ (M-MLV; Promega, Axygen). Negative control reactions were run without reverse transcriptase. An equal volume of product was subjected to PCR. The primer sequences were specific to an updated version of the GenBank sequences. Sequences of primers were used to detect human Lin28 (NM_024674) and let-7a (MIMAT0000062) (GeneChem). The amplification conditions were as follows: 40 cycles of $95^{\circ} \mathrm{C}$ for $30 \mathrm{sec}, 95^{\circ} \mathrm{C}$ for $5 \mathrm{sec}$, annealing at $60^{\circ} \mathrm{C}$ for $30 \mathrm{sec}$ and $72^{\circ} \mathrm{C}$ for $15 \mathrm{sec}$ for primer template extension. Gene expression in each sample was normalized to glyceraldehyde-3-phosphate dehydrogenase (GAPDH) expression. The fold changes for each mRNA were calculated using the $2^{-\triangle \Delta C T}$ method. The different forward (F) and reverse (R) primer sequences were as follows: the primers for Lin28 were (forward) 5'-GCCTTCTACTGGAAGATTGG and (reverse) 5'-GATCTGGAGATGTAAAGCACG; the primers for GAPDH were (forward) 5'-TGACTTCAACAGCGAC ACCCA and (reverse) 5'-CACCCTGTTGCTGTAGCCAAA.

Western blot analysis. Cell proteins were extracted using lysis buffer [50 mM Tris- $\mathrm{HCl}$ (pH 8.8), $150 \mathrm{mM} \mathrm{NaCl,} \mathrm{0.1 \%} \mathrm{SDS,}$ $2 \mathrm{mM}$ EDTA, $1 \mathrm{mM}$ PMSF, $1 \% \mathrm{NP} 40,5 \mu \mathrm{g} / \mathrm{ml}$ aprotinin, $1 \mu \mathrm{g} / \mathrm{ml}$ leupeptin] at $4^{\circ} \mathrm{C}$ on ice. The lysates were centrifuged at $12,000 \mathrm{rpm}$ for $10 \mathrm{~min}$ at $4^{\circ} \mathrm{C}$, and the supernatants were collected. Twenty-five micrograms of protein was loaded onto a $12 \%$ SDS-PAGE gel and subjected to electrophoresis at $20 \mathrm{~mA}$ for 62 min under denaturing conditions and then transferred to nitrocellulose membranes. After blocking in 5\% non-fat milk in Tris-buffered saline Tween-20 (TBST) overnight at $4^{\circ} \mathrm{C}$, the membranes were immunoblotted with anti-Lin 28 $(1: 3,000)$ or anti- $\beta$-actin $(1: 5,000)$ antibodies overnight at $4^{\circ} \mathrm{C}$. The following day, membranes were washed three times with TBST and then incubated with anti-mouse secondary antibodies in PBS $(1: 4,000)$ for $2 \mathrm{~h}$ at room temperature. Bands were detected using an enhanced chemiluminescence (ECL) system (ECL kit; Santa Cruz, USA). $\beta$-actin was used as an internal control.

Cell proliferation assay. An MTT assay was used to evaluate cell proliferation according to the manufacturer's instructions. After recombinant lentivirus transfection for 4 days, the three groups were seeded in 96-well plates at 2,000 cells/well and cultured for $24,48,72,96$ and $120 \mathrm{~h}$. A total of $20 \mu \mathrm{l}$ of MTT (Sigma) stock solution was added to $200 \mu \mathrm{l}$ of medium in each well, and the plates were incubated for $4 \mathrm{~h}$ at $37^{\circ} \mathrm{C}$. Next, 

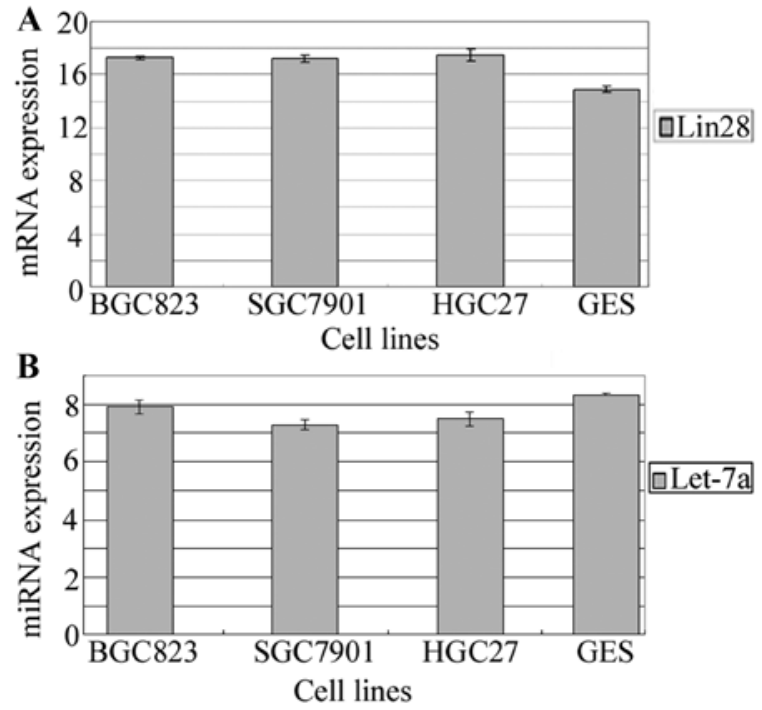

Figure 1. Lin28 and let-7a expression in the cell lines. (A) mRNA was moderately expressed in the GES cells and lowly expressed in the BGC-823, SGC-7901 and HGC-27 cells. (B) Let-7a miRNA was highly expressed in the GES cells and in the gastric cancer cell lines.

$150 \mu \mathrm{l}$ of dimethyl sulfoxide was added to each well, and the plates were incubated for $15 \mathrm{~min}$ at room temperature. The absorbance values were read by an enzyme-linked immunosorbent assay (490 $\mathrm{nm})$.

Migration assay. A Transwell assay was used to evaluate cell migration. After recombinant lentivirus transfection for 4 days, $1 \times 10^{5}$ cells were plated onto 24 -well cell chambers (3422; Corning Inc., Corning, NY, USA) with an $8-\mu$ m pore polycarbonate membrane. In this assay, the cells were plated in medium supplemented with $0.1 \%$ serum, and the chambers were placed into 24 -well plates with medium containing $10 \%$ serum. After $24 \mathrm{~h}$, invaded cells on the lower membrane surface were fixed and stained with Giemsa stain while cells that did not migrate through the pores were removed by cotton swabs. Cells in three random fields for each insert were counted with fluorescence microscopy (Olympus). The absorbance values were read by an enzyme-linked immunosorbent assay $(570 \mathrm{~nm})$.

Cell cycle analysis. To analyze cell cycle distribution, DNA content per duplicate was analyzed using flow cytometry. After recombinant lentivirus transfection for 6 days, the cells in the three groups were harvested by trypsinization, centrifuged and washed three times with $4^{\circ} \mathrm{C}$ PBS. The cells were then fixed in $70 \%$ ethanol at $4^{\circ} \mathrm{C}$ for $1 \mathrm{~h}$. The fixed cells were stained with $50 \mu \mathrm{g} / \mathrm{ml}$ propidium iodide (PI) containing $50 \mu \mathrm{g} /$ $\mathrm{ml}$ RNase A (DNase free) for $15 \mathrm{~min}$ at room temperature in the dark and analyzed by fluorescence-activated cell sorting (FACSCalibur; BD Biosciences, San Jose, CA, USA). The results were analyzed using CellQuest software (BectonDickinson and Co., Franklin Lakes, NJ, USA). The cell cycle distribution was evaluated by calculating the proportion of cells in the G0/G1, S and G2/M stages. In each independent experiment, three parallel wells were contructed, and the procedures were carried out in triplicate.
Apoptosis analysis. The Annexin V-APC apoptosis detection kit (eBioscience Inc., San Diego, CA, USA) was used to evaluate apoptosis. After recombinant lentivirus transfection for 9 days, cell in the three groups were harvested and centrifuged for $5 \mathrm{~min}$ at 1,500 $\mathrm{rmp}$, and then suspended at a density of $1 \times 10^{6}$ cells $/ \mathrm{ml}$. Subsequently, the cells were diluted by buffer, and $5 \mu \mathrm{l}$ Annexin $\mathrm{V}$ was added to each sample. Incubation was carried out for another $15 \mathrm{~min}$, and samples were analyzed using flow cytometry (BD Biosciences) and observed by fluorescence microscopy (Olympus). Data obtained were analyzed using CellQuest software.

Statistical analysis. Statistical analysis was performed with SPSS 13.0 software (version 13.0). The Student's t-test, one-way ANOVA and Pearson's Chi-square test were used according to the data characteristics. $\mathrm{P}<0.05$ was considered to indicate a statistically significant result. The quantitative data are presented as means \pm standard deviation.

\section{Results}

Lin28 and let-7 expression in gastric cancer cell lines. Using real-time PCR to quantify Lin 28 mRNA and hsa-let-7a-5p in four human gastric cancer cell lines and a normal gastric epithelium cell line, we found that Lin 28 mRNA was moderately expressed in the GES cells and expressed at a low level in the BGC-823, SGC-7901 and HGC-27 cells. let-7a miRNA was highly expressed in both GES cells and the gastric cancer cell lines (Fig. 1). The expression of Lin 28 mRNA was inversely correlated with let-7a $(r=-0.727, \mathrm{P}=0.007)$. These results suggest that Lin 28 may play important roles in gastric cancer progression by acting as a tumor-suppressor gene and let-7a may also play an important role.

Lenti-Lin28 upregulates the expression of Lin28 in the $B G C-823$ cells. After lentivirus transduction at $20 \mathrm{MOI}$ for $72 \mathrm{~h}$, the cell viability was $\sim 90$, and $80 \%$ of the BGC- 823 cells steadily delivered the GFP gene (Fig. 2). RT-PCR analysis was performed to detect Lin 28 expression in the BGC- 823 cells. The mRNA level of Lin28 was low or undetectable in the NC group, yet exposure of the BGC-823 cells to lenti-Lin 28 for $72 \mathrm{~h}$ significantly elevated the level of Lin28. The Lin28A mRNA levels in the $\mathrm{OE}$ and $\mathrm{NC}$ groups were 28.5 \pm 2.3 and $104.5 \pm 6.4 \%$, respectively ( $\mathrm{P}=0.0027$ ) (Fig. $3 \mathrm{~A})$. Moreover, to verify whether the increase in Lin $28 \mathrm{mRNA}$ was translated into upregulation of the protein, we assayed the protein expression of Lin 28 in BGC-823 cells. In accordance with the mRNA level, western blotting showed that the OE group expressed a higher protein level following lentivirus Lin28 administration (Fig. 3B).

Overexpression of Lin 28 downregulates let-7a expression in the BGC-823 cells. We assessed let-7a expression in the NC and OE groups with RT-PCR. Let-7a miRNA levels were 100.7 \pm 14.1 and $54.4 \pm 2.5 \%$, respectively (Fig. 3C). The expression level of let-7a miRNA in the OE group was significantly lower than that in the NC group $(\mathrm{P}=0.02647)$. The overexpression of Lin 28 mRNA was inversely correlated with let-7a $(\mathrm{r}=-0.863, \mathrm{P}=0.003)$. Systematic overexpression of Lin28 markedly downregulated the miRNA expression of let-7a. 

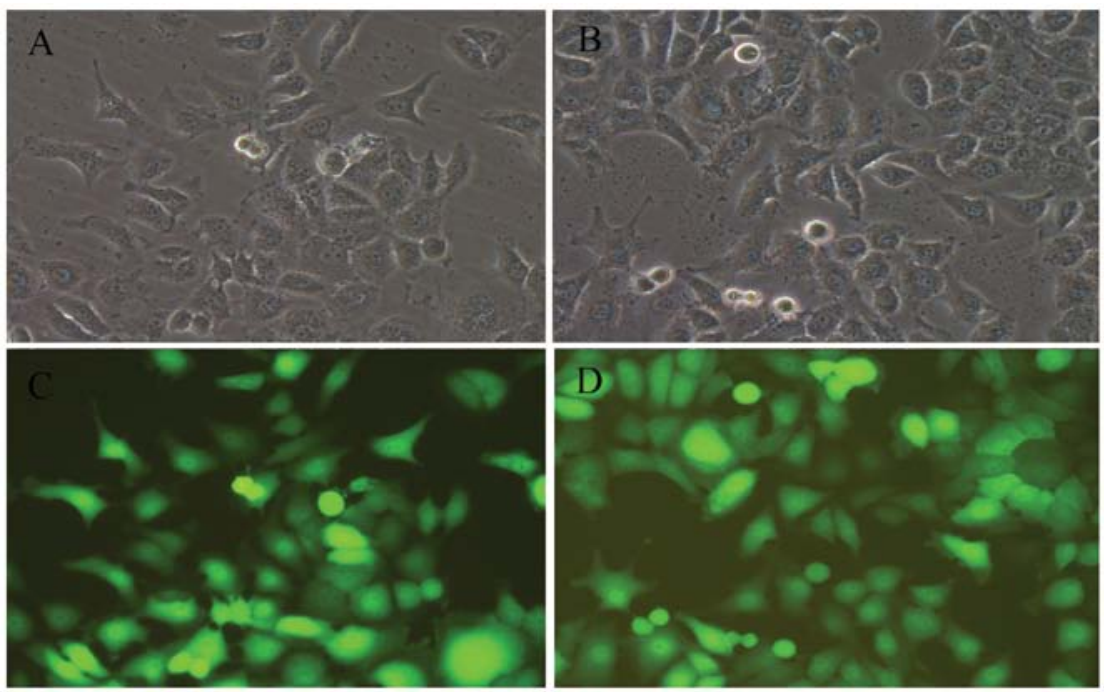

Figure 2. Representative images of BGC-823 cells infected with the indicated lentiviruses. (A) NC group, bright field. (B) OE group, bright field. (C) NC group, fluorescent field. (D) OE group, fluorescent field. Magnification, x200. NC, negative control; OE, overexpression.

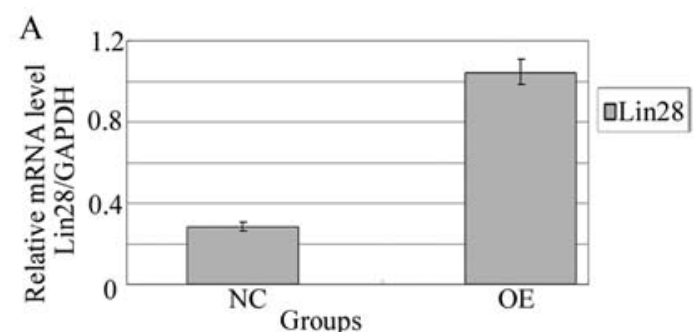

B
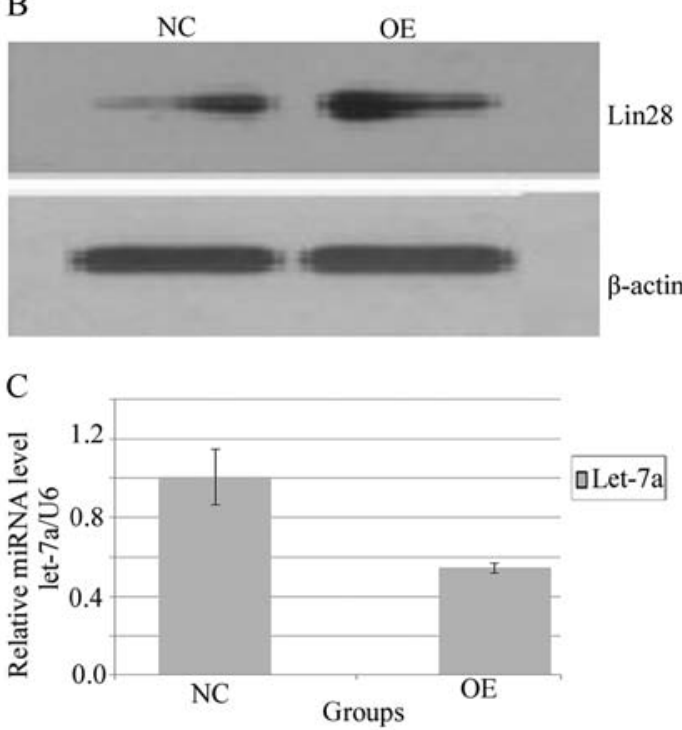

Figure 3. Lin28 and let-7a expression after lenti-Lin28 transduction. (A) The mRNA level of Lin28 was significantly elevated in the OE group and was low or undetectable in the NC group after lenti-Lin28 transduction for $72 \mathrm{~h}$ (B) Western blotting showed that the OE group expressed a higher protein level than that of the NC group. (C) The expression level of let-7a miRNA in the $\mathrm{OE}$ group was significantly lower than that in the NC group. OE, overexpression; NC, negative control.

Overexpression of Lin28 inhibits gastric cancer cell proliferation and migration. An MTT assay was employed to study the impact of overexpression of Lin28 on cell proliferation. The
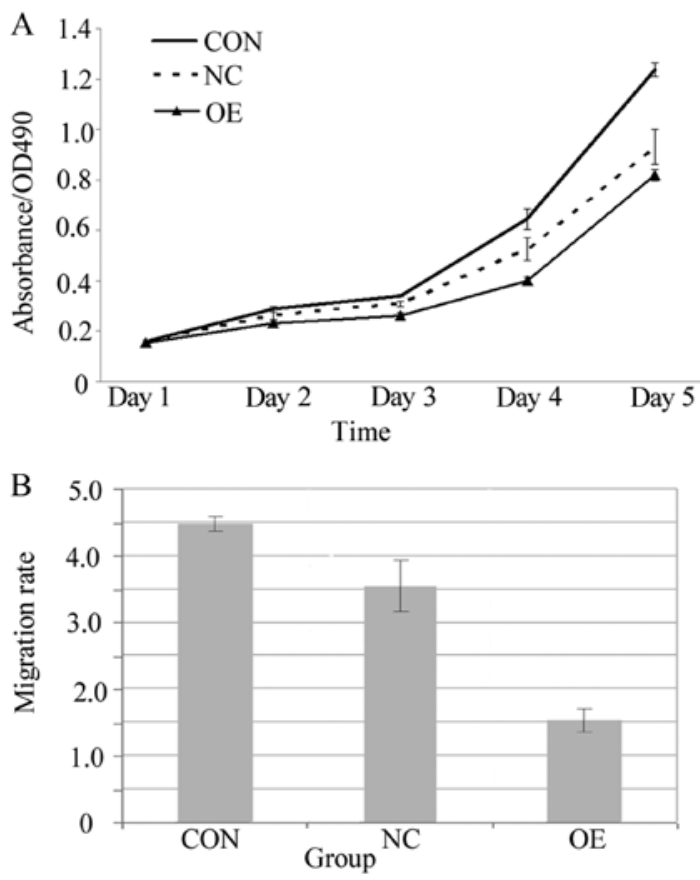

Figure 4. Outcomes of the MTT and Transwell assays. (A) The rate of cell proliferation was slower in the $\mathrm{OE}$ group than the rates in the $\mathrm{CON}$ and $\mathrm{NC}$ groups. (B) The migration rate was significantly decreased in the OE group when compared with the rates in the $\mathrm{CON}$ and $\mathrm{NC}$ groups. OE, overexpression; $\mathrm{CON}$, control; $\mathrm{NC}$, negative control.

number of proliferating cells was determined by measuring the optical density (OD) value at $490 \mathrm{~nm}$. The cell lines in all of the groups were cultured for $144 \mathrm{~h}$, and the OD values were then determined and compared among the groups. The results indicated that the rate of cell proliferation was slower in the OE group than the rates in the $\mathrm{CON}$ and $\mathrm{NC}$ groups $(\mathrm{P}<0.05$, Fig. 4A). A Transwell assay was used to determine the influence of the overexpression of Lin 28 on cell migration capability. Differences in migration were distinctly observed among the three groups after Giemsa staining (Fig. 5). The 


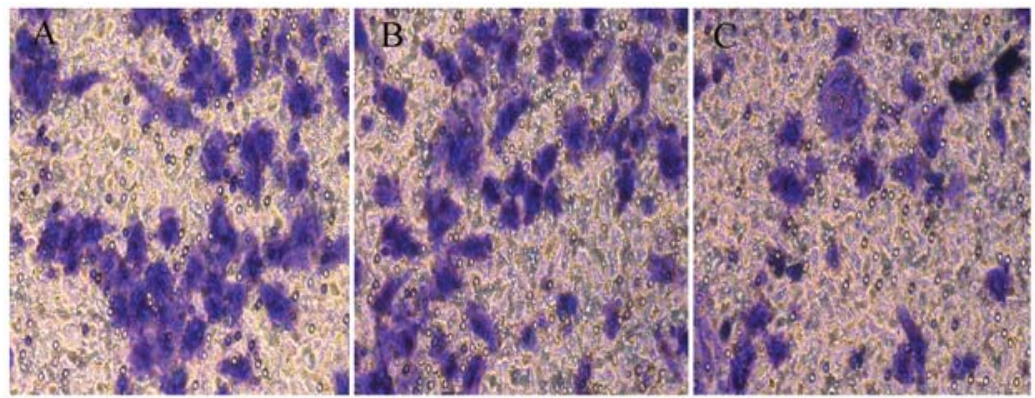

Figure 5. Representative images of Giemsa staining in the different groups. (A) CON group, (B) NC group, (C) OE group. CON, control; NC, negative control; OE, overexpression.
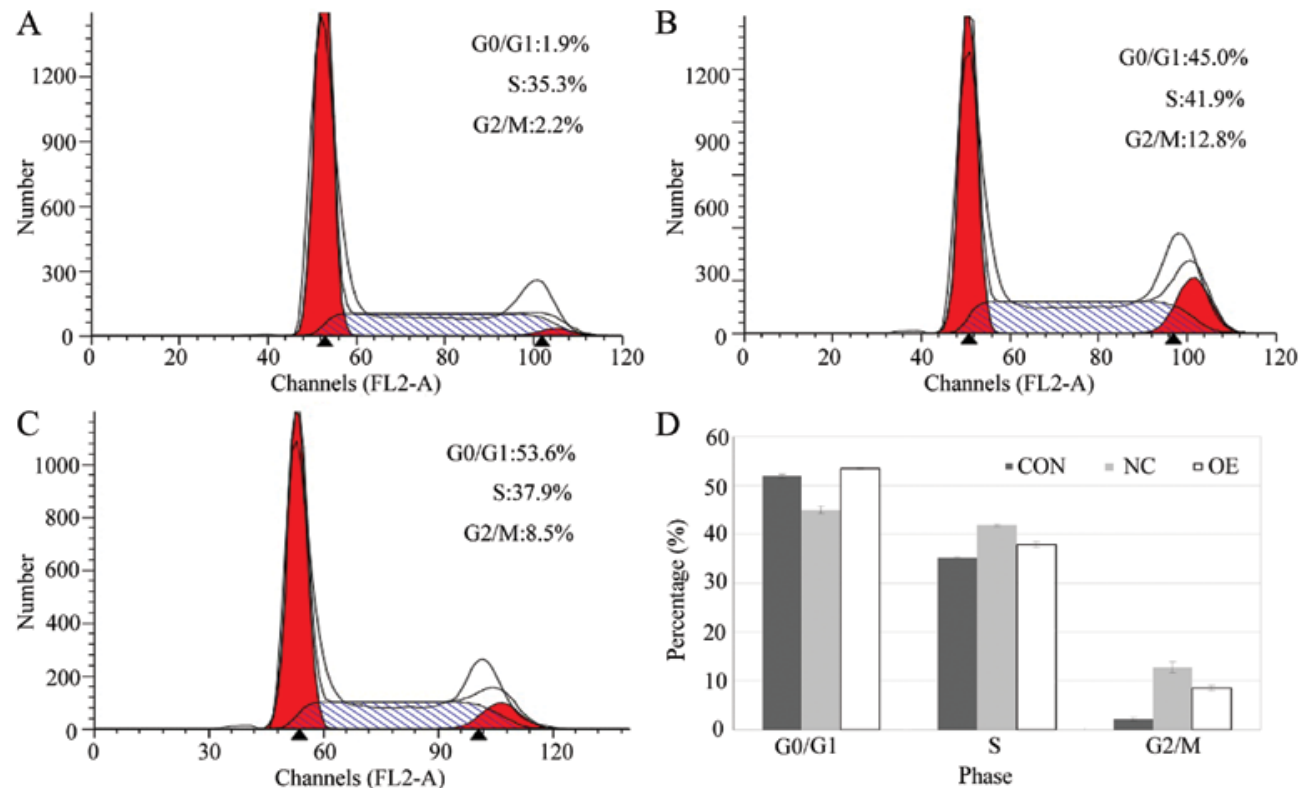

Figure 6. Overexpression of Lin28 inhibits the proliferation of BGC-823 cells due to G0/G1 cell cycle arrest and increased apoptosis. Cell cycle profiles were obtained, and the cell distribution displayed is representative of three groups: (A) control group, (B) NC group, (C) OE group. (D) The percentage of cells in the G0/G1 phase in the OE group was significantly increased while the percentage of cells in the G2/M phase was significantly decreased. NC, negative control; OE, overexpression.
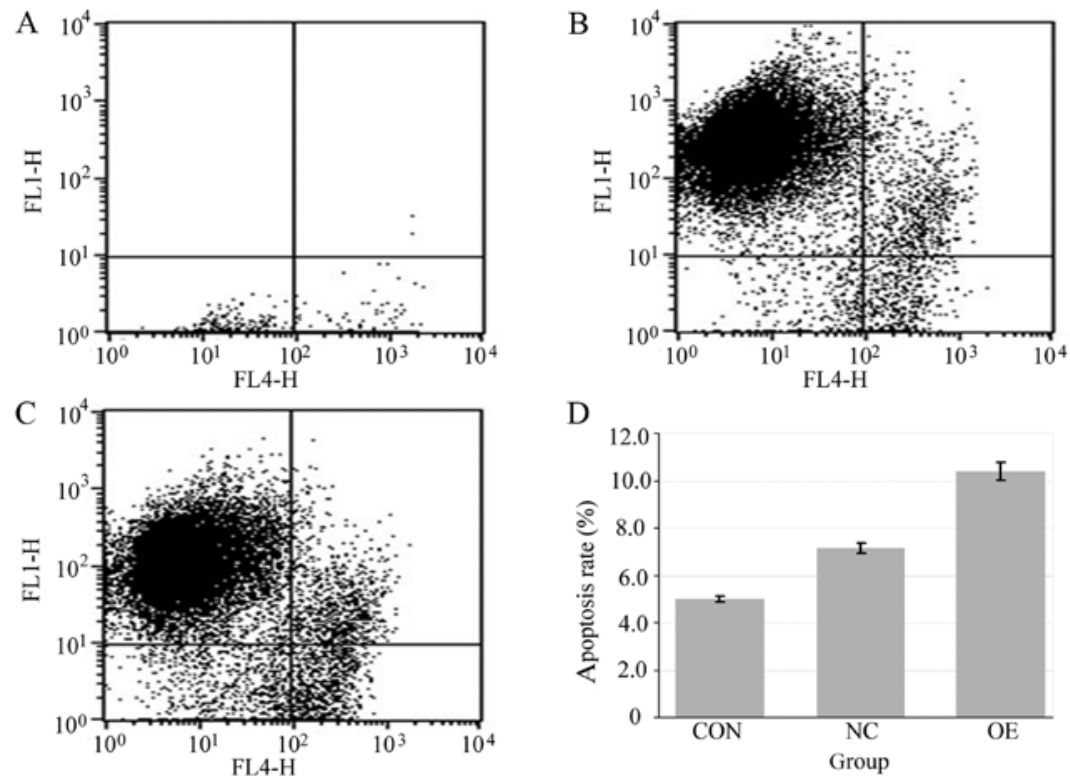

Figure 7. The apoptosis rate was significantly increased in the OE group. Flow cytometry scatter plots were obtained for the three groups: (A) control group, (B) NC group, (C) OE group. (D) The apoptosis rate of the OE group was significantly increased. OE, overexpression; $\mathrm{NC}$, negative control. 
observations revealed that the migration rate was significantly decreased in the OE group when compared with the rates in the $\mathrm{CON}$ and $\mathrm{NC}$ groups $(\mathrm{P}<0.05$, Fig. 4B).

Overexpression of Lin28 affects cell cycle progression and the rate of apoptosis. Cell cycle profiles were monitored by flow cytometric analysis. Compared with that of the CON and NC groups, the percentage of cells in the G0/G1 phase in the OE group was significantly increased, while the percentage of these cells in the G2/M phase was significantly decreased (Fig. 6). These findings indicate that the inhibitory effect on cell proliferation by Lin 28 may be due to G0/G1 cell cycle arrest and increased apoptosis in BGC-823 cells. The rate of apoptosis in the OE group was significantly increased when compared with the rates in the CON and $\mathrm{NC}$ groups (Fig. 7).

\section{Discussion}

In the present study, we investigated the association of Lin28 expression with let-7 in gastric cancer cells. We found that Lin28 expression was downregulated while let-7a was highly expressed in the gastric cancer cell lines, and that Lin28 was inversely correlated with let-7a. Lin 28 transfection induced downregulation of let-7a miRNA and suppression of proliferation, migration and cell cycle progression in the gastric cancer cells. These results suggest that Lin 28 may suppress tumor malignancy of gastric cancer cells through inhibition of let-7 microRNA biogenesis and could be a potential target for gastric cancer therapy.

A possible interference of Lin28 towards let-7 maturation process has been reported both in developmental stages and in various human malignancies. This inverse relationship between Lin28 and let-7 miRNA is present in mammalian cells, where Lin28A/B are mainly expressed in undifferentiated cells, and mature let-7 is only detectable upon differentiation or tissue development $(13,14,17)$.

In undifferentiated cells, Lin 28 is highly expressed and blocks the biogenesis of let-7 miRNA. Upon differentiation, Lin28 expression is reduced and leads to increased levels of mature let-7. Let-7 can silence gene expression of proto-oncogenes (Ras, c-Myc, Hmga2), cell cycle progression factors (cyclin D1 and D3, Cdk4), components of the PI3K-mTOR pathway and Lin28 itself, thereby establishing a positive feedback loop. Besides its role in differentiation, a Lin28/let-7 regulatory network is apparently involved in several cellular processes such as development and physiology, proliferation, oncogenesis, as well as metabolism $(18,19)$. In the present study, our results revealed that an inverse relationship between Lin28 and let-7a miRNA was also present in gastric cancer cells.

The roles of Lin28A and Lin28B are considered to be homogeneous in the oncogenesis of various types of cancer (14), yet the details of the mechanism remain unknown. Several studies have shown that Lin28B is associated with an aggressive biological behavior in several human malignancies $(8,20)$. For instance, Viswanathan et al reported that activation of Lin $28 \mathrm{~B}$ may be associated with rare amplification or translocation of various oncogenes (7). When Lin28B was overexpressed in colon cancer, cellular proliferation in tumor cells was elevated, which then often resulted in metastasis and aggressive tumorigenesis (21). In ovarian cancer, higher expression of Lin28B appears to be linked with poor prognosis of patients possibly via mediation of insulin-like growth factor-II (13).

Research has also highlighted the roles of Lin28 in the biological behavior of gastric cancer. In previous studies, it has been reported that Lin 28 is expressed at a low level in gastric carcinoma tissues when compared with the expression in the corresponding normal tissues, and positive expression of Lin28 protein was found to be correlated with poor outcome. Thus, positive expression of Lin 28 protein serves as an independent prognostic factor (22). Lin28 expression was also associated with pathologic tumor response in locally advanced gastric cancer patients undergoing neoadjuvant chemotherapy and Lin 28 could serve as a predictive biomarker for neoadjuvant chemotherapy in patients with gastric cancer (23). However, in the present study, the results revealed that overexpression of Lin 28 inhibited the proliferation, migration, cell cycle progression and induced the apoptosis of gastric cancer cells, and Lin28 is associated with suppressive biological behavior in gastric cancer cells. To clarify the precise roles of Lin28, further investigation is required including further analysis of the molecular mechanisms.

Collectively, the present study demonstrated that Lin28 inhibited the proliferation, migration and cell cycle progression of gastric cancer cells, while let-7 microRNA may play an important role in this process. We suggest that Lin28 may be a candidate predictor or an anticancer therapeutic target for gastric cancer patients.

\section{Acknowledgements}

This study was supported by Grants from the Open Project of Jiangsu Province Tumor Biological Therapy Institute (no. ZL1203), and the Natural Science Foundation Project of Jiangsu Provincial Education Department (no. 13KJB320029).

\section{References}

1. Moss EG, Lee RC and Ambros V: The cold shock domain protein LIN-28 controls developmental timing in C. elegans and is regulated by the lin-4 RNA. Cell 88: 637-646, 1997.

2. Yu J, Vodyanik MA, Smuga-Otto K, et al: Induced pluripotent stem cell lines derived from human somatic cells. Science 318: 1917-1920, 2007.

3. West JA, Viswanathan SR, Yabuuchi A, et al: A role for $\operatorname{Lin} 28$ in primordial germ-cell development and germ-cell malignancy. Nature 460: 909-913, 2009.

4. Wong SS, Ritner C, Ramachandran S, et al: miR-125b promotes early germ layer specification through Lin28/let-7d and preferential differentiation of mesoderm in human embryonic stem cells. PLoS One 7: e36121, 2012.

5. Gonzalez F, Barragan Monasterio M, Tiscornia G, et al: Generation of mouse-induced pluripotent stem cells by transient expression of a single nonviral polycistronic vector. Proc Natl Acad Sci USA 106: 8918-8922, 2009.

6. Thornton JE and Gregory RI: How does Lin28 let-7 control development and disease? Trends Cell Biol 22: 474-482, 2012.

7. Viswanathan SR, Powers JT, Einhorn W, et al: Lin 28 promotes transformation and is associated with advanced human malignancies. Nat Genet 41: 843-848, 2009.

8. Guo Y, Chen Y, Ito H, Watanabe A, Ge X, Kodama T and Aburatani H: Identification and characterization of lin-28 homolog B (LIN28B) in human hepatocellular carcinoma. Gene 384: 51-61, 2006.

9. Saiki Y, Ishimaru S, Mimori K, et al: Comprehensive analysis of the clinical significance of inducing pluripotent stemness-related gene expression in colorectal cancer cells. Ann Surg Oncol 16: 2638-2644, 2009. 
10. Pan L, Gong Z, Zhong Z, Dong Z, Liu Q, Le Y and Guo J: Lin-28 reactivation is required for let-7 repression and proliferation in human small cell lung cancer cells. Mol Cell Biochem 355: 257-263, 2011.

11. Heo I, Joo C, Cho J, Ha M, Han J and Kim VN: Lin28 mediates the terminal uridylation of let-7 precursor microRNA. Mol Cell 32: 276-284, 2008.

12. Newman MA, Thomson JM and Hammond SM: Lin-28 interaction with the Let-7 precursor loop mediates regulated microRNA processing. RNA 14: 1539-1549, 2008.

13. Rybak A, Fuchs H, Smirnova L, Brandt C, Pohl EE, Nitsch R and Wulczyn FG: A feedback loop comprising lin-28 and let-7 controls pre-let-7 maturation during neural stem-cell commitment. Nat Cell Biol 10: 987-993, 2008.

14. Viswanathan SR, Daley GQ and Gregory RI: Selective blockade of microRNA processing by Lin28. Science 320: 97-100, 2008.

15. Viswanathan SR and Daley GQ: Lin28: a microRNA regulator with a macro role. Cell 140: 445-449, 2010.

16. Thomson JA, Itskovitz-Eldor J, Shapiro SS, Waknitz MA, Swiergiel JJ, Marshall VS and Jones JM: Embryonic stem cell lines derived from human blastocysts. Science 282: 1145-1147, 1998.
17. Darr H and Benvenisty N: Genetic analysis of the role of the reprogramming gene $L I N-28$ in human embryonic stem cells. Stem Cells 27: 352-362, 2009.

18. Thornton JE, Chang HM, Piskounova E and Gregory RI: Lin28-mediated control of let-7 microRNA expression by alternative TUTases Zcchc11 (TUT4) and Zcchc6 (TUT7). RNA 18: 1875-1885, 2012.

19. Mayr F and Heinemann U: Mechanisms of Lin28-mediated miRNA and mRNA regulation - a structural and functional perspective. Int J Mol Sci 14: 16532-16553, 2013.

20. Chang TC, Zeitels LR, Hwang HW, et al: Lin-28B transactivation is necessary for Myc-mediated let-7 repression and proliferation. Proc Natl Acad Sci USA 106: 3384-3389, 2009.

21. King CE, Cuatrecasas M, Castells A, Sepulveda AR, Lee JS and Rustgi AK: LIN28B promotes colon cancer progression and metastasis. Cancer Res 71: 4260-4268, 2011.

22. Xu C, Shen J, Xie S, Jiang Z, Huang L and Wang L: Positive expression of Lin 28 is correlated with poor survival in gastric carcinoma. Med Oncol 30: 382, 2013.

23. Teng RY, Zhou JC, Jiang ZN, et al: The relationship between Lin 28 and the chemotherapy response of gastric cancer. Onco Targets Ther 6: 1341-1345, 2013. 